\title{
13. CARBON AND CARBONATE ANALYSES, LEG 9
}

\author{
R. E. Boyce and G. W. Bode, Scripps Institute of Oceanography
}

The purpose of these analyses is to determine the amount of acid-soluble and insoluble carbon in the sediment samples and to report the results in terms of "calcium carbonate" and "organic" carbon. These data are listed in Table 1, and discussed in Site Report Chapters. Step by step procedures are in the Initial Reports of the Deep Sea Drilling Project, Volume IV, but brief methodology follows with a detailed discussion of calibration procedures and precision.

Each sample was ground to a homogeneous fine powder, dried 24 hours at 105 to $110^{\circ} \mathrm{C}$, divided into two parts, and weighed. The first part was analyzed for its total carbon content and the second part was acidified with hydrochloric acid and then analyzed for residual carbon, which is referred to as "organic" carbon. The carbon contents are reported in terms of per cent by weight, and the theoretical percentages of calcium carbonate in the sediment sample are calculated as follows: $(\%$ total $\mathrm{C}-\% \mathrm{C}$ after acidification $) \times 8.33=$ $\%$ calcium carbonate $\left(\mathrm{CaCO}_{3}\right)$. However, carbonate sediments may not be composed entirely of calcium carbonate, but may include magnesium carbonate, iron carbonate, or some other carbonate, which may result in theoretical calcium-carbonate percentages greater or less than the true compositions.

The carbon determinations were made using a LECO (Laboratory Equipment Corporation) carbon analyzer which is described in detail in the LECO manual:

The LECO 70 Second Carbon Analyzer utilizes the difference in thermal conductivity between oxygen and carbon dioxide. In the method, a sample of known weight is placed in a ceramic crucible to which is added one or more combustion accelerators such as... [copper and iron metal chip]. The crucible containing the sample is then placed in a LECO High Frequency Induction Furnace within a combustion tube through which oxygen is passed.

The carbon in the sample is converted to $\mathrm{CO}_{2}$ at temperatures in excess of $1600^{\circ} \mathrm{C}$. Metal oxides either remain in the crucible or are filtered out in a dust trap while sulfur gases are absorbed in a trap containing manganese dioxide. Any carbon monoxide is converted to $\mathrm{CO}_{2}$ in a heated catalyst tube [containing a mixture of rare earth and copper oxides]. Moisture is removed in an anhydrone [trap].

The carbon dioxide formed and the carrier oxygen are collected in a cylinder. The thermal conductivity of the gas mixtuer contained in the cylinder is measured by a thermistor-type thermal conductivity cell. The output of the thermal conductivity cell is read on a special DC digital voltmeter. With pure oxygen in the cylinder, the thermal conductivity cell is balanced to yield 0.000 output as indicated on the digital voltmeter. With the instrument thus balanced, the output of the thermal conductivity cell is indicated on the digital voltmeter and is proportional to the amount of $\mathrm{CO}_{2}$ in the cylinder.

The cylinder is housed in a temperature controlled oven where the temperature is set above ambient to eliminate temperature variations, which would cause an unbalance of the thermal conductivity cell. ${ }^{1}$

In the conductivity cell two thermistors are placed in small cavities in a metal block. When oxygen in one cavity is replaced by carbon dioxide, which has a lower thermal conductivity than oxygen, that thermistor becomes hotter and lower in electrical resistance. This electrical resistance change is calibrated to read per cent carbon dioxide in oxygen. For detailed electronics of Model 521, see the LECO manual.

The 70 second analyzer was empirically calibrated at the DSDP shore-based laboratory with homogeneous sediment standards whose carbonate content was determined by both a DSDP chemist $^{2}$ (by gravimetrically measuring the $\mathrm{CO}_{2}$ evolved from the sample after treatment with perchloric acid), and by commercial

\footnotetext{
${ }^{1}$ Laboratory Equipment Corporation, St. Joseph, Michigan. Instruction Manual for the LECO 70 Second Carbon Analyzer, Form 146C. pp. 1-2.

${ }^{2}$ Richard DuBois
} 
laboratories. This calibration also agreed with the National Bureau of Standards Argillaceous Limestone Standard Reference Material lb and Dolomite Limestone $88 \mathrm{a}$

Reagent grade calcium-carbonate determinations, however, were slightly low (total carbon low by 0.1 to 0.3 per cent). These results are similar to those of Franklin (1970) who reported values 0.1 to 0.3 per cent low when analyzing reagent grade calcium carbonate with a similar instrument. These low values appear to be related to the very fine grain size of the reagent grade calcium carbonate (van Andel, personal communication).

In order for us to measure carbon content in the sediment standards accurately and precisely with our particular industrial grade oxygen, it became apparent that the amount of copper and iron chip accelerators added to the sample must be carefully controlled. During calibration, the oxygen flow rate was set at 1.5 liters per minute and the LECO carbon analyzer was standardized with LECO Standard iron rings (one ring weighs about 1.0 gram) containing 0.070 and 0.875 per cent carbon to which 1.0 gram of copper chip accelerator (1 flatscoop) was added. Sediment standards were then burned with 1.0 gram of copper and varying weight ( 1.0 to 2.0 grams) of iron chip accelerator, and a direct linear variation in the apparent percentage of $\mathrm{CaCO}_{3}$ was observed. A similar variation was obtained by changing the amount of copper accelerator added to the sample. Variations were also discernible by using a constant amount accelerator with the sample and varying the amount of accelerator added to the standard LECO rings.

The above variations are observed only when using industrial grade oxygen, but not when using ultra high purity oxygen, therefore, it appears that the variation is related to gaseous impurities. As the amount of iron chip is increased it reacts with the oxygen to form iron oxides, thus increasing the relative percentages of the gaseous impurities and introducing a blank which must be corrected for.

The blank was determined by using constant amounts of iron (1.7 grams) and copper (1.0 gram) and different sample weights ( 0.1 to 0.5 grams). These were then processed, and the results varied inversely to the weight of the sample. A constant blank $(0.024)$ was subtracted from the digital readout which then allowed the correct carbon content to be determined regardless of the sample weight. These results agreed very well with the sediment standards and the LECO acid-base system, which is discussed later.

The precision of these data varies because samples within a range of 1.2 to 12 per cent carbon were 0.1 gram samples, while samples within a range of 0 to 1.2 per cent carbon range were 0.5 gram samples.

The precision of the 70 second analyzer data is as follows:

Total carbon

( 1.2 to 12 per cent $)= \pm 0.2 \%$ (absolute variation)

( 0 to 1.2 per cent $)= \pm 0.04 \%$ (absolute variation)

Organic carbon $\quad= \pm 0.04 \%$ (absolute variation)

Calcium carbonate

(10 to 100 per cent $)= \pm 2.0 \%$ (absolute variation)

( 0 to 10 per cent $)= \pm 0.6 \%$ (absolute variation)

With a single operator and over a short period of time the standard deviation for calcium carbonate determinations between 10 and 100 per cent is \pm 0.2 per cent (absolute variation). However, over a long period of time ( 2 months) and several operators the standard deviation is about \pm 0.6 per cent (absolute variation). The standard deviation for calcium carbonate data between 0 and 10 per cent is about \pm 0.15 per cent (absolute variation).

During the processing of Leg 9 samples, the LECO 70 second carbon analyzer became inoperative. At this time, the carbon analysis was conducted using the same furnace but with a different carbon dioxide detection system: A LECO acid-base Semi Automatic Carbon Determinator. With this system the sample is also burned at approximately $1600^{\circ} \mathrm{C}$, but the oxygen flow rate was 0.75 liters per minute. The liberated gas of carbon dioxide and oxygen is volumetrically measured in a solution of dilute sulfuric acid and methyl orange. This gas is then passed through a potassium hydroxide solution which preferentially absorbs carbon dioxide. The volume of the gas is measured a second time. The volume of the carbon dioxide gas is the difference of the two volumetric measurements. The temperature and pressure measurements are taken for the vapor pressure correction of the dilute sulfuric acid solutions, and the volume is then corrected to STP. A similar method is described by Maxwell (1968, p. 227).

The acid-base method obtains correct answers for the National Bureau of Standards rock references and with our own standards. It agrees very well with the 70 second analyzer on the sediment samples and standards. It even obtains good results with reagent grade calcium carbonate, where the 70 second analyzer is slightly lacking. This may be related to the slower oxygen flow rate, which appears to allow a slightly hotter burn and lessens any tendency to blow the sample out of the crucible before it's completely burned.

About 70 samples were processed by the acid-base method and they are indicated in Table 1 by an asterisk adjacent to the carbon percentages. 
TABLE 1

Carbon and Carbonate Analyses, Leg 9

\begin{tabular}{|c|c|c|c|c|c|c|c|}
\hline Hole & Core & Section & $\begin{array}{l}\text { Top Interval in } \\
\text { Section }(\mathrm{cm})\end{array}$ & $\begin{array}{l}\text { Depth in } \\
\text { Hole }(\mathrm{m})\end{array}$ & $\begin{array}{c}\text { Total Carbon } \\
\text { Per Cent }\end{array}$ & $\begin{array}{l}\text { Organic Carbon } \\
\text { Per Cent }\end{array}$ & $\begin{array}{l}\mathrm{CaCO}_{3} \\
\text { Per Cent }\end{array}$ \\
\hline 76 & 1 & 1 & 20.0 & 0.2 & 5.3 & $* 0.1$ & 43 \\
\hline 76 & 1 & 2 & 70.0 & 2.2 & 0.3 & 0.1 & 2 \\
\hline 76 & 1 & 3 & 49.0 & 3.5 & 0.6 & 0.1 & 4 \\
\hline 76 & 1 & 4 & 20.0 & 4.7 & 3.6 & 0.2 & 29 \\
\hline 76 & 1 & 5 & 31.0 & 6.3 & 6.6 & 0.1 & 55 \\
\hline 76 & 1 & 6 & 30.0 & 7.8 & 2.0 & 0.1 & 16 \\
\hline $76 \mathrm{~A}$ & 1 & 2 & 20.0 & 10.8 & 11.0 & 0.1 & 91 \\
\hline $76 \mathrm{~A}$ & 1 & 6 & 90.0 & 17.5 & 11.3 & 0.1 & 94 \\
\hline $76 \mathrm{~A}$ & 2 & 1 & 101.0 & 19.2 & 7.3 & 0.1 & 60 \\
\hline $76 \mathrm{~A}$ & 2 & 3 & 12.0 & 21.3 & 9.8 & 0.1 & 81 \\
\hline $76 \mathrm{~A}$ & 2 & 3 & 86.0 & 22.1 & 10.9 & 0.1 & 90 \\
\hline $76 \mathrm{~A}$ & 2 & 4 & 110.0 & 23.8 & 9.1 & 0.1 & 75 \\
\hline $76 \mathrm{~A}$ & 2 & 5 & 68.0 & 24.9 & 11.2 & 0.1 & 93 \\
\hline $76 \mathrm{~A}$ & 2 & 6 & 80.0 & 26.5 & 10.6 & 0.1 & 87 \\
\hline $76 \mathrm{~A}$ & 2 & 6 & 98.0 & 26.7 & 4.2 & 0.1 & 34 \\
\hline 77 & 1 & 1 & 42.0 & 0.4 & 9.5 & 0.1 & 78 \\
\hline $77 \mathrm{~A}$ & 1 & 1 & 60.0 & 0.6 & 9.1 & 0.1 & 74 \\
\hline $77 \mathrm{~A}$ & 1 & 2 & 30.0 & 1.8 & 9.4 & 0.1 & 77 \\
\hline $77 \mathrm{~A}$ & 1 & 3 & 16.0 & 3.2 & 8.5 & 0.1 & 70 \\
\hline $77 \mathrm{~A}$ & 1 & 4 & 70.0 & 5.2 & 9.8 & 0.3 & 80 \\
\hline $77 \mathrm{~A}$ & 1 & 5 & 71.0 & 6.7 & 9.9 & 0.3 & 80 \\
\hline $77 \mathrm{~A}$ & 1 & 6 & 82.0 & 8.3 & 9.8 & 0.2 & 81 \\
\hline 77B & 1 & 1 & 20.0 & 9.3 & 10.7 & 0.1 & 88 \\
\hline $77 \mathrm{~B}$ & 1 & 2 & 16.0 & 10.8 & 10.0 & 0.2 & 82 \\
\hline 77B & 1 & 3 & 31.0 & 12.4 & 10.0 & 0.2 & 82 \\
\hline $77 \mathrm{~B}$ & 1 & 4 & 17.0 & 13.8 & 10.7 & 0.1 & 88 \\
\hline 77B & 1 & 5 & 102.0 & 16.1 & 10.2 & 0.2 & 83 \\
\hline $77 \mathrm{~B}$ & 1 & 6 & 21.0 & 16.8 & 10.6 & 0.1 & 88 \\
\hline 77B & 2 & 1 & 36.0 & 18.6 & 9.5 & 0.2 & 78 \\
\hline $77 \mathrm{~B}$ & 2 & 2 & 55.0 & 20.3 & 9.3 & 0.1 & 77 \\
\hline $77 \mathrm{~B}$ & 2 & 3 & 20.0 & 21.4 & 10.1 & 0.2 & 83 \\
\hline $77 \mathrm{~B}$ & 2 & 4 & 40.0 & 23.1 & 10.0 & 0.1 & 82 \\
\hline $77 \mathrm{~B}$ & 2 & 5 & 80.0 & 25.0 & 10.1 & 0.1 & 83 \\
\hline $77 \mathrm{~B}$ & 2 & 6 & 35.0 & 26.1 & 9.6 & 0.1 & 79 \\
\hline 77B & 3 & 1 & 35.0 & 27.6 & 9.6 & 0.1 & 79 \\
\hline 77B & 3 & 2 & 23.0 & 29.0 & 9.9 & 0.1 & 82 \\
\hline
\end{tabular}

*These carbon determinations were analyzed with the LECO acid-base Semi Automatic Carbon Determinator Aictom. 
TABLE 1 - Continued

\begin{tabular}{|c|c|c|c|c|c|c|c|}
\hline Hole & Core & Section & $\begin{array}{l}\text { Top Interval in } \\
\text { Section }(\mathrm{cm})\end{array}$ & $\begin{array}{l}\text { Depth in } \\
\text { Hole (m) }\end{array}$ & $\begin{array}{l}\text { Total Carbon } \\
\text { Per Cent }\end{array}$ & $\begin{array}{l}\text { Organic Carbon } \\
\text { Per Cent }\end{array}$ & $\begin{array}{l}\mathrm{CaCO}_{3} \\
\text { Per Cent }\end{array}$ \\
\hline 77B & 3 & 3 & 20.0 & 30.5 & 10.2 & 0.3 & 83 \\
\hline 77B & 3 & 4 & 41.0 & 32.2 & 10.1 & 0.3 & 81 \\
\hline $77 \mathrm{~B}$ & 3 & 5 & 30.0 & 33.6 & 9.6 & 0.0 & 80 \\
\hline 77B & 4 & 1 & 103.0 & 37.6 & 8.9 & 0.3 & 72 \\
\hline $77 \mathrm{~B}$ & 4 & 2 & 24.0 & 38.3 & 9.6 & 0.3 & 78 \\
\hline $77 \mathrm{~B}$ & 4 & 3 & 20.0 & 39.8 & 8.5 & 0.3 & 69 \\
\hline 77B & 4 & 4 & 16.0 & 41.3 & 7.7 & 0.2 & 62 \\
\hline $77 \mathrm{~B}$ & 4 & 5 & 15.0 & 42.8 & 7.5 & 0.3 & 60 \\
\hline $77 \mathrm{~B}$ & 4 & 5 & 92.0 & 43.5 & $* 8.5$ & $*_{0.1}$ & 70 \\
\hline $77 \mathrm{~B}$ & 4 & 6 & 15.0 & 44.3 & 9.1 & 0.2 & 75 \\
\hline $77 \mathrm{~B}$ & 5 & 1 & 16.0 & 45.8 & 8.7 & 0.3 & 70 \\
\hline $77 \mathrm{~B}$ & 5 & 2 & 15.0 & 47.3 & 8.4 & 0.4 & 67 \\
\hline $77 \mathrm{~B}$ & 5 & 3 & 15.0 & 48.8 & 8.4 & 0.3 & 68 \\
\hline $77 \mathrm{~B}$ & 5 & 4 & 16.0 & 50.3 & 8.3 & 0.3 & 66 \\
\hline $77 \mathrm{~B}$ & 5 & 5 & 16.0 & 51.8 & 7.6 & 0.3 & 61 \\
\hline $77 \mathrm{~B}$ & 5 & 6 & 15.0 & 53.3 & 8.3 & 0.2 & 68 \\
\hline $77 \mathrm{~B}$ & 6 & 1 & 16.0 & 55.0 & 9.2 & 0.2 & 75 \\
\hline $77 \mathrm{~B}$ & 6 & 2 & 30.0 & 56.6 & 9.9 & 0.3 & 80 \\
\hline $77 \mathrm{~B}$ & 6 & 3 & 16.0 & 58.0 & 9.1 & 0.3 & 73 \\
\hline $77 \mathrm{~B}$ & 6 & 4 & 16.0 & 59.5 & 7.9 & 0.3 & 64 \\
\hline 77B & 6 & 5 & 16.0 & 61.0 & 8.7 & 0.4 & 70 \\
\hline $77 \mathrm{~B}$ & 6 & 6 & 16.0 & 62.5 & 8.5 & 0.2 & 69 \\
\hline $77 \mathrm{~B}$ & 6 & 6 & 125.0 & 63.5 & $* 10.8$ & $* 0.1$ & 89 \\
\hline 77B & 7 & 1 & 51.0 & 64.5 & 10.5 & 0.2 & 86 \\
\hline $77 \mathrm{~B}$ & 7 & 2 & 16.0 & 65.7 & 10.6 & 0.2 & 86 \\
\hline 77B & 7 & 3 & 16.0 & 67.2 & 9.9 & 0.2 & 81 \\
\hline 77B & 7 & 4 & 16.0 & 68.7 & 10.8 & 0.3 & 88 \\
\hline 77B & 7 & 5 & 16.0 & 70.2 & 9.2 & 0.2 & 75 \\
\hline 77B & 7 & 6 & 97.0 & 72.5 & 9.5 & 0.3 & 76 \\
\hline 77B & 8 & 3 & 16.0 & 76.3 & 9.7 & 0.3 & 78 \\
\hline $77 \mathrm{~B}$ & 8 & 5 & 16.0 & 79.3 & 10.1 & 0.2 & 82 \\
\hline $77 \mathrm{~B}$ & 8 & 6 & 16.0 & 80.8 & 9.9 & 0.2 & 81 \\
\hline $77 \mathrm{~B}$ & 8 & 6 & 125.0 & 81.8 & *9.8 & $* 0.1$ & 82 \\
\hline $77 \mathrm{~B}$ & 9 & 2 & 16.0 & 83.9 & 10.9 & 0.3 & 88 \\
\hline $77 \mathrm{~B}$ & 9 & 4 & 16.0 & 86.9 & 10.8 & 0.5 & 86 \\
\hline 77B & 9 & 6 & 16.0 & 89.9 & 10.8 & 0.3 & 87 \\
\hline $77 \mathrm{~B}$ & 10 & 2 & 16.0 & 93.2 & 11.2 & 0.2 & 91 \\
\hline $77 \mathrm{~B}$ & 10 & 4 & 16.0 & 96.2 & 10.9 & 0.2 & 90 \\
\hline $77 \mathrm{~B}$ & 10 & 6 & 24.0 & 99.2 & 10.9 & 0.2 & 88 \\
\hline
\end{tabular}


TABLE 1 - Continued

\begin{tabular}{|c|c|c|c|c|c|c|c|}
\hline Hole & Core & Section & $\begin{array}{l}\text { Top Interval in } \\
\text { Section }(\mathrm{cm})\end{array}$ & $\begin{array}{l}\text { Depth in } \\
\text { Hole }(\mathrm{m})\end{array}$ & $\begin{array}{l}\text { Total Carbon } \\
\text { Per Cent }\end{array}$ & $\begin{array}{l}\text { Organic Carbon } \\
\text { Per Cent }\end{array}$ & $\begin{array}{l}\mathrm{CaCO}_{3} \\
\text { Per Cent }\end{array}$ \\
\hline 77B & 11 & 1 & 149.0 & 102.1 & 10.2 & 0.1 & 84 \\
\hline $77 \mathrm{~B}$ & 12 & 1 & 15.0 & 109.8 & 11.0 & 0.2 & 90 \\
\hline $77 \mathrm{~B}$ & 12 & 6 & 16.0 & 117.3 & 10.7 & 0.3 & 86 \\
\hline $77 \mathrm{~B}$ & 13 & 2 & 16.0 & 120.5 & 10.1 & 0.2 & 82 \\
\hline $77 \mathrm{~B}$ & 13 & 4 & 15.0 & 123.5 & 10.3 & 0.2 & 85 \\
\hline 77B & 13 & 6 & 16.0 & 126.5 & 10.7 & 0.3 & 87 \\
\hline $77 \mathrm{~B}$ & 14 & 1 & 16.0 & 128.2 & 10.8 & 0.2 & 88 \\
\hline $77 \mathrm{~B}$ & 14 & 2 & 100.0 & 130.5 & $* 8.2$ & $* 0.1$ & 67 \\
\hline 77B & 14 & 4 & 16.0 & 132.7 & 10.1 & 0.3 & 82 \\
\hline $77 \mathrm{~B}$ & 14 & 4 & 106.0 & 133.6 & $* 9.3$ & $* 0.1$ & 77 \\
\hline 77B & 14 & 6 & 16.0 & 135.7 & 9.2 & 0.2 & 75 \\
\hline $77 \mathrm{~B}$ & 14 & 6 & 139.0 & 136.9 & $* 9.0$ & $* 0.1$ & 74 \\
\hline $77 \mathrm{~B}$ & 15 & 2 & 20.0 & 148.8 & 10.6 & 0.3 & 86 \\
\hline $77 \mathrm{~B}$ & 15 & 4 & 16.0 & 151.8 & 11.2 & 0.3 & 91 \\
\hline 77B & 15 & 6 & 16.0 & 154.8 & ${ }^{*} 11.3$ & $* 0.2$ & 93 \\
\hline $77 \mathrm{~B}$ & 15 & 6 & 109.0 & 155.7 & 10.3 & 0.1 & 86 \\
\hline 77B & 16 & 2 & 16.0 & 147.9 & 10.8 & 0.3 & 87 \\
\hline 77B & 16 & 4 & 16.0 & 150.9 & 10.3 & 0.3 & 84 \\
\hline $77 \mathrm{~B}$ & 16 & 6 & 16.0 & 153.9 & $*_{10.1}$ & $* 0.2$ & 82 \\
\hline $77 \mathrm{~B}$ & 17 & 1 & 16.0 & 155.7 & 9.3 & 0.2 & 76 \\
\hline $77 \mathrm{~B}$ & 17 & 2 & 16.0 & 157.2 & 8.6 & 0.2 & 70 \\
\hline 77B & 17 & 2 & 144.0 & 158.4 & $* 8.8$ & $* 0.1$ & 73 \\
\hline 77B & 17 & 6 & 125.0 & 164.3 & $* 7.9$ & $* 0.1$ & 65 \\
\hline 77B & 17 & 4 & 16.0 & 160.2 & 9.9 & 0.1 & 81 \\
\hline 77B & 17 & 6 & 16.0 & 163.2 & 9.6 & 0.1 & 79 \\
\hline 77B & 18 & 2 & 16.0 & 163.3 & 6.5 & 0.2 & 53 \\
\hline 77B & 18 & 2 & 145.0 & 164.6 & $* 9.4$ & $* 0.1$ & 78 \\
\hline 77B & 18 & 4 & 16.0 & 166.3 & 8.2 & 0.2 & 67 \\
\hline $77 \mathrm{~B}$ & 18 & 4 & 125.0 & 167.4 & $* 7.3$ & $* 0.1$ & 59 \\
\hline 77B & 18 & 6 & 16.0 & 169.3 & 7.2 & 0.1 & 59 \\
\hline 77B & 18 & 6 & 123.0 & 170.3 & *9.6 & $* 0.1$ & 79 \\
\hline 77B & 19 & 1 & 35.0 & 171.0 & 8.9 & 0.2 & 72 \\
\hline $77 \mathrm{~B}$ & 19 & 2 & 16.0 & 172.3 & 9.2 & 0.2 & 75 \\
\hline 77B & 19 & 3 & 16.0 & 173.8 & 10.0 & 0.2 & 82 \\
\hline 77B & 19 & 4 & 16.0 & 175.3 & 9.9 & 0.2 & 80 \\
\hline 77B & 19 & 5 & 16.0 & 176.8 & 10.2 & 0.2 & 84 \\
\hline 77B & 19 & 6 & 16.0 & 178.3 & 10.3 & 0.2 & 84 \\
\hline 77B & 20 & 2 & 16.0 & 181.4 & 10.6 & 0.1 & 87 \\
\hline
\end{tabular}


TABLE 1 - Continued

\begin{tabular}{|c|c|c|c|c|c|c|c|}
\hline Hole & Core & Section & $\begin{array}{l}\text { Top Interval in } \\
\text { Section }(\mathrm{cm})\end{array}$ & $\begin{array}{l}\text { Depth in } \\
\text { Hole (m) }\end{array}$ & $\begin{array}{c}\text { Total Carbon } \\
\text { Per Cent }\end{array}$ & $\begin{array}{l}\text { Organic Carbon } \\
\text { Per Cent }\end{array}$ & $\begin{array}{l}\mathrm{CaCO}_{3} \\
\text { Per Cent }\end{array}$ \\
\hline $77 \mathrm{~B}$ & 20 & 2 & 130.0 & 182.5 & $* 11.0$ & $* 0.0$ & 91 \\
\hline $77 \mathrm{~B}$ & 20 & 5 & 16.0 & 185.9 & 10.6 & 0.2 & 87 \\
\hline $77 \mathrm{~B}$ & 20 & 6 & 16.0 & 187.4 & 10.4 & 0.2 & 85 \\
\hline 77B & 21 & 1 & 16.0 & 189.2 & 11.1 & 0.2 & 91 \\
\hline $77 \mathrm{~B}$ & 21 & 2 & 16.0 & 190.7 & 11.4 & 0.2 & 94 \\
\hline 77B & 21 & 2 & 125.0 & 191.8 & $* 11.7$ & $* 0.1$ & 97 \\
\hline $77 \mathrm{~B}$ & 21 & 5 & 16.0 & 195.2 & 11.6 & 0.2 & 95 \\
\hline 77B & 21 & 5 & 77.0 & 195.8 & $* 11.7$ & $* 0.7$ & 92 \\
\hline 77B & 22 & 2 & 16.0 & 199.8 & 10.3 & 0.3 & 84 \\
\hline $77 \mathrm{~B}$ & 22 & 2 & 124.0 & 200.8 & $* 10.6$ & $* 0.0$ & 88 \\
\hline 77B & 22 & 5 & 16.0 & 204.3 & 10.5 & 0.2 & 86 \\
\hline 77B & 22 & 6 & 16.0 & 205.8 & 10.6 & 0.3 & 86 \\
\hline $77 \mathrm{~B}$ & 23 & 2 & 16.0 & 208.9 & 10.9 & 0.2 & 89 \\
\hline $77 \mathrm{~B}$ & 23 & 2 & 125.0 & 209.9 & $* 10.5$ & $* 0.5$ & 84 \\
\hline 77B & 23 & 5 & 16.0 & 213.4 & 11.4 & 0.2 & 93 \\
\hline $77 \mathrm{~B}$ & 23 & 6 & 16.0 & 214.9 & 11.2 & 0.2 & 92 \\
\hline $77 \mathrm{~B}$ & 24 & 2 & 16.0 & 218.0 & 11.6 & 0.2 & 94 \\
\hline 77B & 24 & 2 & 124.0 & 219.0 & $* 11.1$ & $* 0.1$ & 92 \\
\hline 77B & 24 & 5 & 30.0 & 222.6 & 10.9 & 0.3 & 89 \\
\hline $77 \mathrm{~B}$ & 24 & 6 & 16.0 & 224.0 & 11.2 & 0.3 & 91 \\
\hline 77B & 24 & 6 & 139.0 & 225.2 & $*_{10.8}$ & $* 0.3$ & 87 \\
\hline $77 \mathrm{~B}$ & 25 & 2 & 16.0 & 227.3 & 11.4 & 0.3 & 93 \\
\hline $77 \mathrm{~B}$ & 25 & 2 & 141.0 & 228.5 & $* 11.2$ & $* 0.0$ & 93 \\
\hline $77 \mathrm{~B}$ & 25 & 4 & 15.0 & 230.3 & $* 11.2$ & $* 0.0$ & 93 \\
\hline $77 \mathrm{~B}$ & 25 & 5 & 16.0 & 231.8 & 10.2 & 0.3 & 82 \\
\hline $77 \mathrm{~B}$ & 25 & 6 & 16.0 & 233.3 & 10.9 & 0.2 & 89 \\
\hline $77 \mathrm{~B}$ & 26 & 2 & 35.0 & 236.5 & 11.3 & 0.3 & 92 \\
\hline $77 \mathrm{~B}$ & 26 & 2 & 139.0 & 237.5 & $* 10.8$ & $* 0.1$ & 90 \\
\hline 77B & 26 & 5 & 16.0 & 240.8 & 11.1 & 0.3 & 90 \\
\hline $77 \mathrm{~B}$ & 26 & 6 & 16.0 & 242.3 & 11.0 & 0.2 & 90 \\
\hline 77B & 26 & 6 & 125.0 & 243.4 & $*_{11.2}$ & $* 0.0$ & 93 \\
\hline $77 \mathrm{~B}$ & 27 & 1 & 22.0 & 244.0 & 9.3 & 0.4 & 74 \\
\hline $77 \mathrm{~B}$ & 27 & 2 & 30.0 & 245.6 & 8.6 & 0.4 & 68 \\
\hline $77 \mathrm{~B}$ & 27 & 5 & 16.0 & 250.0 & 9.4 & 0.3 & 76 \\
\hline $77 \mathrm{~B}$ & 27 & 6 & 16.0 & 251.5 & 9.7 & 0.3 & 78 \\
\hline $77 \mathrm{~B}$ & 28 & 2 & 46.0 & 255.0 & 10.1 & 0.2 & 82 \\
\hline 77B & 28 & 5 & 27.0 & 259.3 & 9.8 & 0.3 & 80 \\
\hline $77 \mathrm{~B}$ & 28 & 6 & 16.0 & 260.7 & 10.4 & 0.4 & 84 \\
\hline $77 \mathrm{~B}$ & 29 & 2 & 22.0 & 263.8 & 11.1 & 0.2 & 91 \\
\hline
\end{tabular}


TABLE 1 - Continued

\begin{tabular}{|c|c|c|c|c|c|c|c|}
\hline Hole & Core & Section & $\begin{array}{l}\text { Top Interval in } \\
\text { Section }(\mathrm{cm})\end{array}$ & $\begin{array}{l}\text { Depth in } \\
\text { Hole }(\mathrm{m})\end{array}$ & $\begin{array}{c}\text { Total Carbon } \\
\text { Per Cent }\end{array}$ & $\begin{array}{l}\text { Organic Carbon } \\
\text { Per Cent }\end{array}$ & $\begin{array}{l}\mathrm{CaCO}_{3} \\
\text { Per Cent }\end{array}$ \\
\hline 77B & 29 & 5 & 16.0 & 268.3 & 11.1 & 0.2 & 91 \\
\hline 77B & 29 & 6 & 16.0 & 269.8 & 11.1 & 0.3 & 90 \\
\hline $77 \mathrm{~B}$ & 30 & 2 & 16.0 & 272.9 & 11.0 & 0.3 & 89 \\
\hline $77 \mathrm{~B}$ & 30 & 5 & 16.0 & 277.4 & $*_{10.9}$ & 0.2 & 90 \\
\hline 77B & 30 & 6 & 15.0 & 278.9 & 11.6 & 0.1 & 95 \\
\hline $77 \mathrm{~B}$ & 31 & 2 & 25.0 & 282.0 & 11.5 & 0.2 & 94 \\
\hline 77B & 31 & 5 & 16.0 & 286.5 & 11.9 & 0.1 & 98 \\
\hline $77 \mathrm{~B}$ & 31 & 6 & 16.0 & 288.0 & 11.5 & 0.1 & 94 \\
\hline 77B & 32 & 2 & 16.0 & 291.3 & 11.6 & 0.2 & 95 \\
\hline $77 \mathrm{~B}$ & 32 & 5 & 16.0 & 295.8 & 11.5 & 0.1 & 95 \\
\hline $77 \mathrm{~B}$ & 32 & 6 & 16.0 & 297.3 & 11.3 & 0.1 & 93 \\
\hline 77B & 33 & 2 & 14.0 & 300.2 & 11.3 & 0.2 & 93 \\
\hline $77 \mathrm{~B}$ & 33 & 5 & 14.0 & 304.7 & 11.4 & 0.2 & 93 \\
\hline $77 \mathrm{~B}$ & 33 & 5 & 14.0 & 304.7 & 11.4 & 0.1 & 94 \\
\hline $77 \mathrm{~B}$ & 33 & 6 & 16.0 & 306.3 & 11.8 & 0.1 & 97 \\
\hline 77B & 34 & 2 & 16.0 & 309.5 & 11.5 & 0.3 & 94 \\
\hline $77 \mathrm{~B}$ & 34 & 5 & 16.0 & 314.0 & 11.6 & 0.2 & 95 \\
\hline $77 \mathrm{~B}$ & 34 & 6 & 16.0 & 315.5 & 11.7 & 0.2 & 96 \\
\hline $77 \mathrm{~B}$ & 36 & 2 & 16.0 & 327.8 & 11.2 & 0.2 & 92 \\
\hline $77 \mathrm{~B}$ & 36 & 3 & 16.0 & 329.3 & 11.5 & 0.1 & 95 \\
\hline 77B & 37 & 2 & 16.0 & 336.9 & 11.3 & 0.1 & 93 \\
\hline $77 \mathrm{~B}$ & 37 & 5 & 16.0 & 341.4 & 11.0 & 0.1 & 90 \\
\hline $77 \mathrm{~B}$ & 37 & 6 & 16.0 & 342.9 & 11.2 & 0.1 & 93 \\
\hline $77 \mathrm{~B}$ & 38 & 2 & 82.0 & 346.6 & 11.3 & 0.2 & 93 \\
\hline 77B & 39 & 5 & 16.0 & 359.8 & 11.4 & 0.2 & 93 \\
\hline 77B & 39 & 6 & 16.0 & 361.3 & 12.2 & 0.1 & 100 \\
\hline $77 \mathrm{~B}$ & 40 & 2 & 16.0 & 364.3 & 11.8 & 0.5 & 94 \\
\hline $77 \mathrm{~B}$ & 40 & 3 & 20.0 & 365.8 & 11.2 & 0.5 & 89 \\
\hline 77B & 40 & 4 & 16.0 & 367.3 & 11.7 & 0.1 & 97 \\
\hline $77 \mathrm{~B}$ & 41 & 2 & 16.0 & 373.5 & 11.5 & 0.1 & 95 \\
\hline 77B & 41 & 5 & 16.0 & 378.0 & 11.7 & 0.1 & 97 \\
\hline $77 \mathrm{~B}$ & 41 & 6 & 16.0 & 379.5 & 11.6 & 0.1 & 96 \\
\hline $77 \mathrm{~B}$ & 42 & 2 & 16.0 & 382.7 & 11.5 & 0.1 & 95 \\
\hline $77 \mathrm{~B}$ & 42 & 5 & 20.0 & 387.2 & 11.8 & 0.1 & 97 \\
\hline $77 \mathrm{~B}$ & 42 & 6 & 16.0 & 388.7 & 11.7 & 0.1 & 97 \\
\hline 77B & 43 & 2 & 16.0 & 391.8 & 11.4 & 0.1 & 94 \\
\hline $77 \mathrm{~B}$ & 43 & 5 & 16.0 & 396.3 & 11.4 & 0.1 & 94 \\
\hline $77 \mathrm{~B}$ & 43 & 6 & 16.0 & 397.8 & 11.6 & 0.1 & 96 \\
\hline $77 \mathrm{~B}$ & 44 & 2 & 16.0 & 400.9 & 11.6 & 0.1 & 96 \\
\hline
\end{tabular}


TABLE 1 - Continued

\begin{tabular}{|c|c|c|c|c|c|c|c|}
\hline Hole & Core & Section & $\begin{array}{l}\text { Top Interval in } \\
\text { Section }(\mathrm{cm})\end{array}$ & $\begin{array}{l}\text { Depth in } \\
\text { Hole }(m)\end{array}$ & $\begin{array}{l}\text { Total Carbon } \\
\text { Per Cent }\end{array}$ & $\begin{array}{l}\text { Organic Carbon } \\
\text { Per Cent }\end{array}$ & $\begin{array}{l}\mathrm{CaCO}_{3} \\
\text { Per Cent }\end{array}$ \\
\hline 77B & 44 & 5 & 15.0 & 405.4 & 11.6 & 0.1 & 96 \\
\hline $77 \mathrm{~B}$ & 44 & 6 & 15.0 & 406.9 & 11.8 & 0.1 & 97 \\
\hline $77 \mathrm{~B}$ & 45 & 2 & 16.0 & 410.0 & 11.5 & 0.1 & 95 \\
\hline $77 \mathrm{~B}$ & 45 & 5 & 16.0 & 414.5 & 11.7 & 0.1 & 97 \\
\hline $77 \mathrm{~B}$ & 45 & 6 & 16.0 & 416.0 & 11.5 & 0.1 & 95 \\
\hline 77B & 46 & 2 & 16.0 & 419.3 & 11.3 & 0.1 & 94 \\
\hline $77 \mathrm{~B}$ & 46 & 5 & 16.0 & 423.8 & 11.5 & 0.1 & 95 \\
\hline $77 \mathrm{~B}$ & 46 & 6 & 16.0 & 425.3 & 11.6 & 0.1 & 96 \\
\hline 77B & 47 & 2 & 16.0 & 428.3 & 11.1 & 0.1 & 92 \\
\hline 77B & 47 & 4 & 21.0 & 431.3 & 8.7 & 0.1 & 72 \\
\hline $77 \mathrm{~B}$ & 48 & 2 & 19.0 & 437.5 & 10.8 & 0.1 & 89 \\
\hline $77 \mathrm{~B}$ & 48 & 5 & 16.0 & 442.0 & 10.2 & 0.1 & 84 \\
\hline $77 \mathrm{~B}$ & 48 & 6 & 22.0 & 443.5 & 11.0 & 0.1 & 91 \\
\hline $77 \mathrm{~B}$ & 49 & 2 & 17.0 & 446.7 & 11.0 & 0.1 & 90 \\
\hline $77 \mathrm{~B}$ & 49 & 5 & 45.0 & 451.5 & 11.3 & 0.2 & 93 \\
\hline $77 \mathrm{~B}$ & 49 & 6 & 16.0 & 452.7 & 11.5 & 0.1 & 94 \\
\hline 77B & 50 & 2 & 11.0 & 455.7 & 11.4 & 0.2 & 94 \\
\hline 77B & 51 & 5 & 16.0 & 469.4 & 11.5 & 0.1 & 95 \\
\hline 77B & 51 & 6 & 16.0 & 470.9 & 11.5 & 0.1 & 94 \\
\hline $77 \mathrm{~B}$ & 52 & 1 & 82.0 & 471.6 & 5.2 & 0.4 & 41 \\
\hline $77 \mathrm{~B}$ & 52 & 2 & 16.0 & 472.5 & 1.0 & 0.6 & 3 \\
\hline $77 \mathrm{~B}$ & 53 & 1 & 62.0 & 476.9 & 5.9 & 0.2 & 48 \\
\hline 78 & 1 & 2 & 16.0 & 1.7 & 10.1 & 0.2 & 82 \\
\hline 78 & 1 & 4 & 16.0 & 4.7 & 10.6 & 0.2 & 87 \\
\hline 78 & 1 & 6 & 16.0 & 7.7 & 10.7 & 0.0 & 89 \\
\hline 78 & 2 & 2 & 16.0 & 10.8 & 10.5 & 0.2 & 86 \\
\hline 78 & 2 & 4 & 16.0 & 13.8 & 10.4 & 0.2 & 85 \\
\hline 78 & 2 & 6 & 16.0 & 16.8 & 10.1 & 0.3 & 82 \\
\hline 78 & 3 & 2 & 16.0 & 19.9 & 10.5 & 0.4 & 85 \\
\hline 78 & 3 & 4 & 24.0 & 22.9 & 10.2 & 0.1 & 84 \\
\hline 78 & 3 & 6 & 27.0 & 26.0 & 10.3 & 0.3 & 84 \\
\hline 78 & 4 & 2 & 16.0 & 29.1 & 8.1 & 0.3 & 65 \\
\hline 78 & 4 & 4 & 16.0 & 32.1 & 7.6 & 0.4 & 60 \\
\hline 78 & 4 & 6 & 16.0 & 35.1 & 9.1 & 0.3 & 74 \\
\hline 78 & 5 & 2 & 0.0 & 38.1 & 9.4 & 0.2 & 76 \\
\hline 78 & 5 & 4 & 22.0 & 41.3 & 9.6 & 0.2 & 78 \\
\hline 78 & 5 & 6 & 16.0 & 44.3 & 9.4 & 0.1 & 77 \\
\hline 78 & 6 & 2 & 16.0 & 47.4 & 9.7 & 0.2 & 79 \\
\hline
\end{tabular}


TABLE 1 - Continued

\begin{tabular}{|c|c|c|c|c|c|c|c|}
\hline Hole & Core & Section & $\begin{array}{l}\text { Top Interval in } \\
\text { Section }(\mathrm{cm})\end{array}$ & $\begin{array}{l}\text { Depth in } \\
\text { Hole }(\mathrm{m})\end{array}$ & $\begin{array}{l}\text { Total Carbon } \\
\text { Per Cent }\end{array}$ & $\begin{array}{l}\text { Organic Carbon } \\
\text { Per Cent }\end{array}$ & $\begin{array}{l}\mathrm{CaCO}_{3} \\
\text { Per Cent }\end{array}$ \\
\hline 78 & 6 & 4 & 16.0 & 50.4 & 10.3 & 0.2 & 84 \\
\hline 78 & 6 & 6 & 16.0 & 53.4 & 10.8 & 0.2 & 88 \\
\hline 78 & 7 & 2 & 16.0 & 56.6 & 10.8 & 0.1 & 89 \\
\hline 78 & 7 & 4 & 16.0 & 59.6 & 11.1 & 0.2 & 91 \\
\hline 78 & 7 & 5 & 16.0 & 61.1 & 11.2 & 0.1 & 92 \\
\hline 78 & 8 & 1 & 75.0 & 64.8 & 10.1 & 0.3 & 81 \\
\hline 78 & 9 & 6 & 78.0 & 81.4 & 11.2 & 0.1 & 92 \\
\hline 78 & 10 & 2 & 16.0 & 84.0 & 11.3 & 0.1 & 93 \\
\hline 78 & 10 & 4 & 16.0 & 87.0 & 11.2 & 0.1 & 93 \\
\hline 78 & 10 & 6 & 16.0 & 90.0 & 11.2 & 0.2 & 92 \\
\hline 78 & 11 & 2 & 16.0 & 93.1 & 11.0 & 0.2 & 91 \\
\hline 78 & 11 & 4 & 16.0 & 96.1 & 11.3 & 0.1 & 93 \\
\hline 78 & 11 & 6 & 16.0 & 99.1 & 11.4 & 0.1 & 93 \\
\hline 78 & 12 & 4 & 16.0 & 105.3 & 11.1 & 0.2 & 91 \\
\hline 78 & 12 & 6 & 16.0 & 108.3 & 11.3 & 0.2 & 93 \\
\hline 78 & 13 & 2 & 6.0 & 111.3 & 11.0 & 0.2 & 90 \\
\hline 78 & 13 & 4 & 16.0 & 114.4 & 11.4 & 0.1 & 94 \\
\hline 78 & 13 & 6 & 16.0 & 117.4 & 11.0 & 0.2 & 90 \\
\hline 78 & 14 & 4 & 19.0 & 123.6 & 11.2 & 0.2 & 91 \\
\hline 78 & 14 & 6 & 16.0 & 126.6 & 10.5 & 0.3 & 85 \\
\hline 78 & 15 & 4 & 16.0 & 132.7 & 11.3 & 0.2 & 93 \\
\hline 78 & 15 & 6 & 16.0 & 135.7 & 10.3 & 0.3 & 83 \\
\hline 78 & 16 & 4 & 16.0 & 141.8 & 11.2 & 0.1 & 92 \\
\hline 78 & 16 & 6 & 16.0 & 144.8 & 10.0 & 0.2 & 81 \\
\hline 78 & 17 & 2 & 16.0 & 148.0 & 11.2 & 0.2 & 92 \\
\hline 78 & 17 & 4 & 16.0 & 151.0 & 10.7 & 0.2 & 88 \\
\hline 78 & 17 & 6 & 54.0 & 154.3 & 11.1 & 0.2 & 91 \\
\hline 78 & 19 & 4 & 34.0 & 169.4 & 11.1 & 0.2 & 91 \\
\hline 78 & 19 & 6 & 23.0 & 172.3 & 10.9 & 0.2 & 90 \\
\hline 78 & 20 & 2 & 16.0 & 175.4 & 10.5 & 0.3 & 85 \\
\hline 78 & 20 & 4 & 16.0 & 178.4 & 11.1 & 0.2 & 90 \\
\hline 78 & 20 & 6 & 23.0 & 181.4 & 11.1 & 0.2 & 90 \\
\hline 78 & 21 & 2 & 12.0 & 184.5 & 10.7 & 0.3 & 87 \\
\hline 78 & 21 & 4 & 16.0 & 187.6 & 10.9 & 0.2 & 89 \\
\hline 78 & 21 & 6 & 16.0 & 190.6 & 10.9 & 0.2 & 89 \\
\hline 78 & 22 & 6 & 22.0 & 199.9 & 11.0 & 0.2 & 90 \\
\hline 78 & 23 & 4 & 16.0 & 205.9 & 8.0 & 0.2 & 65 \\
\hline 78 & 23 & 6 & 16.0 & 208.9 & 8.0 & 2.7 & 44 \\
\hline 78 & 24 & 2 & 35.0 & 212.2 & 11.2 & 0.3 & 91 \\
\hline
\end{tabular}


TABLE 1 - Continued

\begin{tabular}{|c|c|c|c|c|c|c|c|}
\hline Hole & Core & Section & $\begin{array}{l}\text { Top Interval in } \\
\text { Section }(\mathrm{cm})\end{array}$ & $\begin{array}{l}\text { Depth in } \\
\text { Hole }(\mathrm{m})\end{array}$ & $\begin{array}{l}\text { Total Carbon } \\
\text { Per Cent }\end{array}$ & $\begin{array}{c}\text { Organic Carbon } \\
\text { Per Cent }\end{array}$ & $\begin{array}{l}\mathrm{CaCO}_{3} \\
\text { Per Cent }\end{array}$ \\
\hline 78 & 25 & 4 & 16.0 & 224.1 & 11.7 & 0.2 & 96 \\
\hline 78 & 25 & 6 & 16.0 & 227.1 & 11.3 & 0.2 & 92 \\
\hline 78 & 27 & 4 & 56.0 & 242.8 & 11.4 & 0.2 & 94 \\
\hline 78 & 27 & 6 & 16.0 & 245.4 & 11.7 & 0.1 & 97 \\
\hline 78 & 28 & 4 & 45.0 & 251.8 & 11.3 & 0.1 & 93 \\
\hline 78 & 28 & 6 & 2.0 & 254.4 & 11.3 & 0.1 & 94 \\
\hline 78 & 29 & 6 & 16.0 & 263.7 & 11.4 & 0.1 & 94 \\
\hline 78 & 30 & 6 & 16.0 & 272.8 & 11.6 & 0.1 & 96 \\
\hline 78 & 31 & 6 & 16.0 & 282.0 & 11.3 & 0.2 & 93 \\
\hline 78 & 32 & 6 & 16.0 & 291.2 & 10.3 & 0.2 & 84 \\
\hline 78 & 35 & 6 & 77.0 & 319.2 & 9.1 & 0.3 & 73 \\
\hline 79 & 1 & 2 & 16.0 & 1.8 & 6.9 & 0.3 & 55 \\
\hline 79 & 1 & 3 & 16.0 & 3.3 & 7.8 & 0.3 & 63 \\
\hline 79 & 1 & 4 & 16.0 & 4.8 & 10.6 & 0.1 & 88 \\
\hline 79 & 1 & 5 & 16.0 & 6.3 & 9.8 & 0.3 & 80 \\
\hline 79 & 1 & 6 & 16.0 & 7.8 & 7.8 & 0.3 & 63 \\
\hline 79 & 2 & 1 & 16.0 & 60.5 & 7.8 & 0.4 & 62 \\
\hline 79 & 2 & 2 & 16.0 & 62.0 & 8.0 & 0.2 & 66 \\
\hline 79 & 2 & 3 & 16.0 & 63.5 & 7.8 & 0.2 & 64 \\
\hline 79 & 2 & 4 & 16.0 & 65.0 & 7.1 & 0.3 & 56 \\
\hline 79 & 2 & 5 & 16.0 & 66.5 & 6.9 & 0.3 & 55 \\
\hline 79 & 2 & 6 & 16.0 & 68.0 & 9.5 & 0.1 & 78 \\
\hline 79 & 3 & 1 & 16.0 & 127.0 & 9.6 & 0.2 & 78 \\
\hline 79 & 3 & 2 & 20.0 & 128.5 & 8.9 & 0.2 & 72 \\
\hline 79 & 3 & 3 & 16.0 & 130.0 & 9.0 & 0.2 & 73 \\
\hline 79 & 3 & 4 & 16.0 & 131.5 & 9.2 & 0.2 & 75 \\
\hline 79 & 3 & 5 & 16.0 & 133.0 & 9.3 & 0.2 & 76 \\
\hline 79 & 4 & 1 & 16.0 & 193.7 & 9.3 & 0.2 & 76 \\
\hline 79 & 4 & 2 & 16.0 & 195.2 & 9.2 & 0.2 & 75 \\
\hline 79 & 4 & 3 & 16.0 & 196.7 & 8.3 & 0.1 & 68 \\
\hline 79 & 4 & 4 & 16.0 & 198.2 & 8.4 & 0.2 & 68 \\
\hline 79 & 4 & 5 & 16.0 & 199.7 & 9.4 & 0.2 & 77 \\
\hline 79 & 4 & 6 & 16.0 & 201.2 & 8.9 & 0.2 & 72 \\
\hline 79 & 5 & 3 & 38.0 & 263.7 & 10.9 & 0.2 & 90 \\
\hline 79 & 5 & 4 & 30.0 & 265.1 & 11.0 & 0.2 & 90 \\
\hline 79 & 5 & 5 & 16.0 & 266.5 & 10.3 & 0.2 & 85 \\
\hline 79 & 5 & 6 & 16.0 & 268.0 & 9.4 & 0.2 & 77 \\
\hline 79 & 6 & 1 & 114.0 & 327.8 & 6.5 & 0.2 & 52 \\
\hline
\end{tabular}


TABLE 1 - Continued

\begin{tabular}{|c|c|c|c|c|c|c|c|}
\hline Hole & Core & Section & $\begin{array}{l}\text { Top Interval in } \\
\text { Section }(\mathrm{cm})\end{array}$ & $\begin{array}{l}\text { Depth in } \\
\text { Hole }(m)\end{array}$ & $\begin{array}{l}\text { Total Carbon } \\
\text { Per Cent }\end{array}$ & $\begin{array}{l}\text { Organic Carbon } \\
\text { Per Cent }\end{array}$ & $\begin{array}{l}\mathrm{CaCO}_{3} \\
\text { Per Cent }\end{array}$ \\
\hline 79 & 6 & 2 & 16.0 & 328.4 & 8.1 & 0.2 & 66 \\
\hline 79 & 6 & 3 & 16.0 & 329.9 & 7.1 & 0.2 & 57 \\
\hline 79 & 6 & 4 & 16.0 & 331.4 & 9.6 & 0.1 & 78 \\
\hline 79 & 6 & 5 & 16.0 & 332.9 & 8.8 & 0.2 & 72 \\
\hline 79 & 7 & 1 & 30.0 & 336.2 & 10.0 & 0.2 & 82 \\
\hline 79 & 7 & 2 & 16.0 & 337.6 & 9.2 & 0.2 & 76 \\
\hline 79 & 7 & 3 & 16.0 & 339.1 & 8.7 & 0.2 & 71 \\
\hline 79 & 7 & 4 & 18.0 & 340.6 & 9.8 & 0.2 & 80 \\
\hline 79 & 7 & 5 & 16.0 & 342.1 & 9.6 & 0.2 & 79 \\
\hline 79 & 8 & 1 & 16.0 & 345.2 & 8.5 & 0.3 & 68 \\
\hline 79 & 8 & 3 & 16.0 & 348.2 & 9.3 & 0.2 & 76 \\
\hline 79 & 8 & 4 & 16.0 & 349.7 & 9.4 & 0.2 & 76 \\
\hline 79 & 8 & 5 & 18.0 & 351.2 & 6.4 & 0.3 & 51 \\
\hline 79 & 8 & 6 & 16.0 & 352.7 & 9.5 & 0.2 & 78 \\
\hline 79 & 9 & 1 & 16.0 & 350.3 & 10.7 & 0.2 & 88 \\
\hline 79 & 9 & 2 & 16.0 & 351.8 & 10.7 & 0.2 & 88 \\
\hline 79 & 9 & 3 & 16.0 & 353.3 & 10.7 & 0.1 & 88 \\
\hline 79 & 10 & 2 & 16.0 & 357.4 & 11.0 & 0.1 & 90 \\
\hline 79 & 10 & 3 & 16.0 & 358.9 & 10.9 & 0.2 & 90 \\
\hline 79 & 10 & 4 & 16.0 & 360.4 & 10.8 & 0.1 & 90 \\
\hline 79 & 11 & 2 & 42.0 & 366.1 & 10.8 & 0.1 & 89 \\
\hline 79 & 11 & 3 & 16.0 & 367.4 & 11.3 & 0.1 & 94 \\
\hline 79 & 11 & 4 & 16.0 & 368.9 & $* 10.9$ & 0.1 & 90 \\
\hline 79 & 11 & 5 & 16.0 & 370.4 & 11.1 & 0.1 & 92 \\
\hline 79 & 11 & 6 & 16.0 & 371.9 & 11.3 & 0.0 & 94 \\
\hline 79 & 12 & 1 & 39.0 & 373.8 & 11.1 & 0.1 & 92 \\
\hline 79 & 12 & 2 & 16.0 & 375.1 & 10.7 & 0.0 & 89 \\
\hline 79 & 12 & 3 & 19.0 & 376.6 & 10.8 & 0.1 & 90 \\
\hline 79 & 12 & 4 & 16.0 & 378.1 & 10.3 & 0.1 & 85 \\
\hline 79 & 12 & 5 & 16.0 & 379.6 & 10.5 & 0.1 & 87 \\
\hline 79 & 12 & 6 & 16.0 & 381.1 & 10.4 & 0.1 & 86 \\
\hline 79 & 13 & 2 & 93.0 & 382.8 & 10.8 & 0.1 & 89 \\
\hline 79 & 13 & 3 & 16.0 & 383.6 & 11.0 & 0.1 & 91 \\
\hline 79 & 13 & 4 & 15.0 & 385.0 & 10.8 & 0.1 & 90 \\
\hline 79 & 13 & 5 & 16.0 & 386.6 & 10.7 & 0.0 & 89 \\
\hline 79 & 13 & 6 & 16.0 & 388.1 & 11.1 & 0.0 & 92 \\
\hline 79 & 14 & 1 & 16.0 & 389.7 & 11.1 & 0.1 & 92 \\
\hline 79 & 14 & 2 & 16.0 & 391.2 & 10.6 & 0.1 & 88 \\
\hline 79 & 14 & 6 & 16.0 & 397.2 & 10.5 & 0.0 & 87 \\
\hline
\end{tabular}


TABLE 1 - Continued

\begin{tabular}{|c|c|c|c|c|c|c|c|}
\hline Hole & Core & Section & $\begin{array}{l}\text { Top Interval in } \\
\text { Section }(\mathrm{cm})\end{array}$ & $\begin{array}{l}\text { Depth in } \\
\text { Hole }(\mathrm{m})\end{array}$ & $\begin{array}{c}\text { Total Carbon } \\
\text { Per Cent }\end{array}$ & $\begin{array}{l}\text { Organic Carbon } \\
\text { Per Cent }\end{array}$ & $\begin{array}{c}\mathrm{CaCO}_{3} \\
\text { Per Cent }\end{array}$ \\
\hline 79 & 15 & 2 & 16.0 & 400.4 & 11.1 & 0.0 & 92 \\
\hline 79 & 16 & 3 & 16.0 & 409.2 & 11.5 & 0.4 & 93 \\
\hline $79 \mathrm{~A}$ & 1 & 1 & 56.0 & 9.7 & 7.8 & 0.2 & 63 \\
\hline $79 A$ & 1 & 2 & 16.0 & 10.8 & 5.3 & 0.2 & 43 \\
\hline $79 \mathrm{~A}$ & 1 & 3 & 16.0 & 12.3 & 6.4 & 0.1 & 53 \\
\hline $79 \mathrm{~A}$ & 1 & 4 & 16.0 & 13.8 & 5.5 & 0.1 & 45 \\
\hline $79 \mathrm{~A}$ & 1 & 5 & 16.0 & 15.3 & 4.5 & 0.1 & 36 \\
\hline $79 \mathrm{~A}$ & 2 & 1 & 16.0 & 69.7 & 5.1 & 0.1 & 41 \\
\hline $79 \mathrm{~A}$ & 2 & 2 & 16.0 & 71.2 & 6.7 & 0.1 & 55 \\
\hline $79 A$ & 2 & 3 & 16.0 & 72.7 & 6.8 & 0.1 & 56 \\
\hline $79 \mathrm{~A}$ & 2 & 4 & 16.0 & 74.2 & 7.2 & 0.1 & 59 \\
\hline $79 A$ & 2 & 5 & 16.0 & 75.7 & 7.3 & 0.1 & 60 \\
\hline $79 \mathrm{~A}$ & 2 & 6 & 16.0 & 77.2 & $* 7.1$ & 0.1 & 58 \\
\hline $79 \mathrm{~A}$ & 3 & 1 & 16.0 & 145.3 & 6.5 & 0.1 & 53 \\
\hline $79 \mathrm{~A}$ & 3 & 2 & 16.0 & 146.8 & 6.0 & 0.1 & 49 \\
\hline $79 \mathrm{~A}$ & 3 & 3 & 16.0 & 148.3 & 5.4 & 0.1 & 45 \\
\hline $79 \mathrm{~A}$ & 3 & 4 & 16.0 & 149.8 & 7.1 & 0.1 & 58 \\
\hline $79 \mathrm{~A}$ & 3 & 5 & 16.0 & 151.3 & 8.0 & 0.1 & 66 \\
\hline $79 A$ & 3 & 6 & 16.0 & 152.8 & 5.7 & 0.1 & 47 \\
\hline $79 A$ & 4 & 6 & 16.0 & 286.3 & 9.3 & 0.1 & 77 \\
\hline 80 & 1 & 1 & 16.0 & 0.2 & 9.5 & 0.1 & 78 \\
\hline 80 & 1 & 3 & 16.0 & 3.2 & 9.9 & 0.1 & 82 \\
\hline 80 & 1 & 4 & 16.0 & 4.7 & 10.3 & 0.1 & 85 \\
\hline 80 & 1 & 5 & 16.0 & 6.2 & 9.3 & 0.1 & 77 \\
\hline 80 & 1 & 6 & 16.0 & 7.7 & 10.2 & 0.1 & 85 \\
\hline 80 & 2 & 1 & 15.0 & 61.2 & 8.5 & 0.1 & 70 \\
\hline 80 & 2 & 2 & 15.0 & 62.7 & 8.8 & 0.1 & 73 \\
\hline 80 & 2 & 3 & 15.0 & 64.2 & 8.3 & 0.1 & 68 \\
\hline 80 & 2 & 4 & 15.0 & 65.7 & 8.4 & 0.1 & 69 \\
\hline 80 & 2 & 5 & 15.0 & 67.2 & 3.9 & 0.2 & 31 \\
\hline 80 & 2 & 6 & 15.0 & 68.7 & 7.5 & 0.3 & 61 \\
\hline 80 & 3 & 1 & 16.0 & 127.7 & 10.6 & 0.0 & 88 \\
\hline 80 & 3 & 2 & 16.0 & 129.2 & 9.2 & 0.1 & 77 \\
\hline 80 & 3 & 3 & 16.0 & 130.7 & 10.0 & 0.1 & 83 \\
\hline 80 & 3 & 4 & 16.0 & 132.2 & 10.2 & 0.0 & 84 \\
\hline 80 & 3 & 5 & 16.0 & 133.7 & 9.8 & 0.0 & 81 \\
\hline 80 & 3 & 6 & 16.0 & 135.2 & 9.3 & 0.1 & 77 \\
\hline 80 & 4 & 2 & 16.0 & 167.4 & 11.1 & 0.1 & 92 \\
\hline
\end{tabular}


TABLE 1 - Continued

\begin{tabular}{|c|c|c|c|c|c|c|c|}
\hline Hole & Core & Section & $\begin{array}{l}\text { Top Interval in } \\
\text { Section }(\mathrm{cm})\end{array}$ & $\begin{array}{l}\text { Depth in } \\
\text { Hole }(m)\end{array}$ & $\begin{array}{c}\text { Total Carbon } \\
\text { Per Cent }\end{array}$ & $\begin{array}{l}\text { Organic Carbon } \\
\text { Per Cent }\end{array}$ & $\begin{array}{c}\mathrm{CaCO}_{3} \\
\text { Per Cent }\end{array}$ \\
\hline 80 & 4 & 3 & 16.0 & 168.9 & 11.1 & 0.0 & 92 \\
\hline 80 & 4 & 4 & 16.0 & 170.4 & 10.9 & 0.1 & 91 \\
\hline 80 & 4 & 4 & 27.0 & 170.5 & 9.0 & 0.1 & 75 \\
\hline 80 & 4 & 5 & 16.0 & 171.9 & 11.0 & 0.1 & 91 \\
\hline 80 & 5 & 1 & 40.0 & 193.7 & 9.7 & 0.3 & 79 \\
\hline 80 & 5 & 2 & 16.0 & 195.0 & 8.0 & 0.0 & 66 \\
\hline 80 & 5 & 3 & 16.0 & 196.5 & 10.0 & 0.1 & 83 \\
\hline $80 \mathrm{~A}$ & 2 & 1 & 16.0 & 42.9 & 7.9 & 0.2 & 64 \\
\hline $80 \mathrm{~A}$ & 2 & 2 & 16.0 & 44.4 & 8.2 & 0.2 & 67 \\
\hline $80 \mathrm{~A}$ & 2 & 3 & 16.0 & 45.9 & 7.2 & 0.2 & 59 \\
\hline $80 \mathrm{~A}$ & 2 & 4 & 16.0 & 47.4 & 6.7 & 0.2 & 54 \\
\hline $80 \mathrm{~A}$ & 3 & 1 & 16.0 & 86.8 & 0.3 & 0.1 & 2 \\
\hline $80 \mathrm{~A}$ & 3 & 2 & 16.0 & 88.3 & 6.1 & 0.1 & 50 \\
\hline $80 \mathrm{~A}$ & 3 & 3 & 16.0 & 89.8 & 9.4 & 0.1 & 78 \\
\hline $80 \mathrm{~A}$ & 3 & 4 & 16.0 & 91.3 & 9.0 & 0.0 & 74 \\
\hline $80 \mathrm{~A}$ & 3 & 5 & 16.0 & 92.8 & 9.1 & 0.0 & 75 \\
\hline $80 \mathrm{~A}$ & 4 & 1 & 0.0 & 109.1 & 10.8 & 0.1 & 89 \\
\hline $80 \mathrm{~A}$ & 4 & 2 & 0.0 & 110.6 & 10.2 & 0.0 & 85 \\
\hline $80 \mathrm{~A}$ & 4 & 3 & 0.0 & 112.1 & 10.4 & 0.0 & 87 \\
\hline $80 \mathrm{~A}$ & 4 & 5 & 0.0 & 115.1 & 10.3 & 0.0 & 85 \\
\hline $80 \mathrm{~A}$ & 4 & 6 & 0.0 & 116.6 & 10.0 & 0.0 & 83 \\
\hline $80 \mathrm{~A}$ & 5 & 1 & 110.0 & 147.7 & 9.9 & 0.1 & 82 \\
\hline $80 \mathrm{~A}$ & 5 & 2 & 146.0 & 149.6 & 10.9 & 0.0 & 91 \\
\hline $80 \mathrm{~A}$ & 5 & 3 & 16.0 & 149.8 & 10.0 & 0.1 & 83 \\
\hline $80 \mathrm{~A}$ & 5 & 4 & 16.0 & 151.3 & 10.6 & 0.1 & 88 \\
\hline $80 \mathrm{~A}$ & 5 & 5 & 16.0 & 152.8 & 10.8 & 0.0 & 89 \\
\hline $80 \mathrm{~A}$ & 5 & 6 & 16.0 & 154.3 & 10.6 & 0.1 & 88 \\
\hline 81 & 1 & 1 & 16.0 & 0.2 & 10.2 & 0.1 & 84 \\
\hline 81 & 1 & 2 & 16.0 & 1.7 & 10.5 & 0.1 & 86 \\
\hline 81 & 1 & 4 & 16.0 & 4.7 & 10.6 & 0.1 & 88 \\
\hline 81 & 1 & 5 & 26.0 & 6.3 & 10.2 & 0.1 & 84 \\
\hline 81 & 1 & 6 & 16.0 & 7.7 & 10.6 & 0.1 & 88 \\
\hline 81 & 2 & 2 & 16.0 & 321.4 & 9.4 & 0.1 & 77 \\
\hline 81 & 2 & 4 & 16.0 & 324.4 & 8.4 & 0.1 & 69 \\
\hline 81 & 2 & 5 & 16.0 & 325.9 & 9.5 & 0.1 & 78 \\
\hline 81 & 2 & 6 & 20.0 & 327.4 & 9.0 & 0.1 & 74 \\
\hline 81 & 3 & 2 & 22.0 & 378.0 & 10.0 & 0.2 & 82 \\
\hline 81 & 3 & 3 & 16.0 & 379.5 & 10.2 & 0.1 & 84 \\
\hline
\end{tabular}


TABLE 1 - Continued

\begin{tabular}{|c|c|c|c|c|c|c|c|}
\hline Hole & Core & Section & $\begin{array}{l}\text { Top Interval in } \\
\text { Section }(\mathrm{cm})\end{array}$ & $\begin{array}{l}\text { Depth in } \\
\text { Hole }(m)\end{array}$ & $\begin{array}{c}\text { Total Carbon } \\
\text { Per Cent }\end{array}$ & $\begin{array}{l}\text { Organic Carbon } \\
\text { Per Cent }\end{array}$ & $\begin{array}{l}\mathrm{CaCO}_{3} \\
\text { Per Cent }\end{array}$ \\
\hline 81 & 3 & 5 & 16.0 & 382.5 & 10.2 & 0.1 & 84 \\
\hline 81 & 3 & 6 & 16.0 & 384.0 & 10.0 & 0.1 & 83 \\
\hline 81 & 4 & 1 & 21.0 & 389.3 & 11.4 & 0.3 & 94 \\
\hline 81 & 4 & 5 & 16.0 & 395.3 & 10.4 & 0.1 & 86 \\
\hline 81 & 5 & 3 & 16.0 & 398.8 & 9.6 & 0.1 & 80 \\
\hline 81 & 6 & 3 & 16.0 & 407.9 & 9.5 & 0.3 & 77 \\
\hline 82 & 1 & 1 & 16.0 & 0.2 & 10.4 & 0.4 & 84 \\
\hline 82 & 1 & 2 & 16.0 & 1.7 & 9.5 & 0.2 & 77 \\
\hline 82 & 1 & 3 & 16.0 & 3.2 & 9.7 & 0.2 & 79 \\
\hline 82 & 1 & 4 & 16.0 & 4.7 & 9.6 & 0.2 & 79 \\
\hline 82 & 1 & 5 & 22.0 & 6.2 & 9.8 & 0.1 & 80 \\
\hline 82 & 1 & 6 & 16.0 & 7.7 & 9.5 & 0.1 & 78 \\
\hline 82 & 2 & 1 & 16.0 & 69.0 & 9.8 & 0.1 & 80 \\
\hline 82 & 2 & 2 & 25.0 & 70.6 & 9.1 & 0.1 & 75 \\
\hline 82 & 2 & 3 & 16.0 & 72.0 & 9.6 & 0.1 & 79 \\
\hline 82 & 2 & 4 & 16.0 & 73.5 & 10.1 & 0.1 & 83 \\
\hline 82 & 2 & 5 & 16.0 & 75.0 & 8.8 & 0.1 & 73 \\
\hline 82 & 2 & 6 & 16.0 & 76.5 & 9.0 & 0.1 & 74 \\
\hline 82 & 3 & 1 & 16.0 & 135.5 & 9.6 & 0.1 & 79 \\
\hline 82 & 3 & 2 & 16.0 & 137.0 & 6.8 & 0.3 & 54 \\
\hline 82 & 3 & 3 & 20.0 & 138.5 & 7.1 & 0.2 & 58 \\
\hline 82 & 3 & 4 & 16.0 & 140.0 & 8.3 & 0.2 & 68 \\
\hline 82 & 3 & 5 & 16.0 & 141.5 & 10.0 & 0.1 & 83 \\
\hline 82 & 3 & 6 & 16.0 & 143.0 & 8.8 & 0.1 & 73 \\
\hline 82 & 4 & 1 & 16.0 & 191.5 & 8.2 & 0.2 & 67 \\
\hline 82 & 4 & 3 & 16.0 & 194.5 & 9.2 & 0.2 & 76 \\
\hline 82 & 4 & 4 & 28.0 & 196.1 & 9.8 & 0.2 & 80 \\
\hline 82 & 4 & 5 & 16.0 & 197.5 & 7.4 & 0.1 & 61 \\
\hline 82 & 5 & 2 & 16.0 & 202.2 & 9.8 & 0.1 & 80 \\
\hline 82 & 6 & 3 & 24.0 & 212.8 & 8.5 & 0.1 & 70 \\
\hline 82 & 6 & 4 & 16.0 & 214.3 & 7.9 & 0.1 & 65 \\
\hline 82 & 6 & 5 & 16.0 & 215.8 & 8.4 & 0.1 & 70 \\
\hline 82 & 6 & 6 & 16.0 & 217.3 & 9.2 & 0.2 & 75 \\
\hline $82 \mathrm{~A}$ & 1 & 2 & 16.0 & 20.0 & 9.8 & 0.1 & 80 \\
\hline $82 \mathrm{~A}$ & 1 & 3 & 16.0 & 21.5 & 8.8 & 0.1 & 72 \\
\hline $82 \mathrm{~A}$ & 1 & 4 & 16.0 & 23.0 & 8.9 & 0.1 & 73 \\
\hline $82 \mathrm{~A}$ & 1 & 5 & 16.0 & 24.5 & 10.1 & 0.1 & 84 \\
\hline $82 \mathrm{~A}$ & 1 & 6 & 16.0 & 26.0 & 10.2 & 0.1 & 84 \\
\hline
\end{tabular}


TABLE 1 - Continued

\begin{tabular}{|c|c|c|c|c|c|c|c|}
\hline Hole & Core & Section & $\begin{array}{l}\text { Top Interval in } \\
\text { Section }(\mathrm{cm})\end{array}$ & $\begin{array}{l}\text { Depth in } \\
\text { Hole }(m)\end{array}$ & $\begin{array}{c}\text { Total Carbon } \\
\text { Per Cent }\end{array}$ & $\begin{array}{l}\text { Organic Carbon } \\
\text { Per Cent }\end{array}$ & $\begin{array}{l}\mathrm{CaCO}_{3} \\
\text { Per Cent }\end{array}$ \\
\hline $82 \mathrm{~A}$ & 2 & 1 & 16.0 & 36.7 & 7.5 & 0.2 & 61 \\
\hline $82 \mathrm{~A}$ & 2 & 2 & 16.0 & 38.2 & 9.1 & 0.1 & 75 \\
\hline $82 \mathrm{~A}$ & 2 & 3 & 16.0 & 39.7 & 9.1 & 0.1 & 75 \\
\hline $82 \mathrm{~A}$ & 2 & 4 & 16.0 & 41.2 & 9.3 & 0.1 & 77 \\
\hline $82 \mathrm{~A}$ & 2 & 5 & 16.0 & 42.7 & 9.2 & 0.1 & 76 \\
\hline $82 \mathrm{~A}$ & 2 & 6 & 16.0 & 44.2 & 9.2 & 0.1 & 75 \\
\hline $82 \mathrm{~A}$ & 3 & 1 & 30.0 & 102.0 & 10.7 & 0.1 & 88 \\
\hline $82 \mathrm{~A}$ & 3 & 2 & 15.0 & 103.3 & 10.4 & 0.1 & 86 \\
\hline $82 \mathrm{~A}$ & 3 & 3 & 15.0 & 104.8 & 10.0 & 0.1 & 83 \\
\hline $82 \mathrm{~A}$ & 3 & 4 & 15.0 & 106.3 & 9.6 & 0.1 & 79 \\
\hline $82 \mathrm{~A}$ & 3 & 5 & 15.0 & 107.8 & 10.0 & 0.1 & 82 \\
\hline 83 & 1 & 2 & 16.0 & 2.0 & 9.5 & 0.4 & 76 \\
\hline 83 & 1 & 2 & 82.0 & 2.6 & $* 5.0$ & $* 0.2$ & 41 \\
\hline 83 & 1 & 3 & 16.0 & 3.5 & 5.4 & 0.2 & 43 \\
\hline 83 & 1 & 4 & 16.0 & 5.0 & 5.9 & 0.2 & 48 \\
\hline 83 & 2 & 1 & 16.0 & 5.3 & 6.4 & 0.2 & 52 \\
\hline 83 & 2 & 2 & 16.0 & 6.8 & 6.0 & 0.2 & 49 \\
\hline 83 & 2 & 2 & 89.0 & 7.5 & $* 5.9$ & $* 0.3$ & 47 \\
\hline 83 & 2 & 3 & 16.0 & 8.3 & 9.5 & 0.1 & 78 \\
\hline 83 & 2 & 4 & 16.0 & 9.8 & 8.2 & 0.2 & 67 \\
\hline 83 & 2 & 5 & 16.0 & 11.3 & 8.1 & 0.1 & 66 \\
\hline 83 & 2 & 5 & 74.0 & 11.8 & $* 8.1$ & $* 0.1$ & 67 \\
\hline 83 & 2 & 6 & 16.0 & 12.8 & 3.7 & 0.2 & 29 \\
\hline 83 & 2 & 6 & 124.0 & 13.8 & $* 7.0$ & $* 0.3$ & 55 \\
\hline 83 & 3 & 2 & 16.0 & 16.0 & 6.1 & 0.2 & 50 \\
\hline 83 & 3 & 3 & 16.0 & 17.5 & 5.9 & 0.2 & 47 \\
\hline 83 & 4 & 3 & 16.0 & 72.0 & 5.2 & 0.2 & 42 \\
\hline 83 & 5 & 2 & 16.0 & 137.9 & 7.7 & 0.1 & 63 \\
\hline 83 & 5 & 3 & 16.0 & 139.4 & 8.4 & 0.1 & 69 \\
\hline 83 & 5 & 4 & 16.0 & 140.9 & 6.4 & 0.1 & 53 \\
\hline 83 & 5 & 5 & 16.0 & 142.4 & 6.8 & 0.2 & 55 \\
\hline 83 & 5 & 6 & 16.0 & 143.9 & 6.5 & 0.2 & 52 \\
\hline 83 & 6 & 2 & 16.0 & 204.0 & 8.0 & 0.1 & 66 \\
\hline 83 & 6 & 3 & 16.0 & 205.5 & 7.8 & 0.1 & 64 \\
\hline 83 & 6 & 4 & 16.0 & 207.0 & 8.4 & 0.1 & 69 \\
\hline 83 & 6 & 5 & 16.0 & 208.5 & 9.0 & 0.1 & 74 \\
\hline 83 & 6 & 6 & 16.0 & 210.0 & 8.5 & 0.1 & 70 \\
\hline 83 & 7 & 1 & 100.0 & 222.8 & $* 5.9$ & $* 0.2$ & 48 \\
\hline
\end{tabular}


TABLE 1 - Continued

\begin{tabular}{|c|c|c|c|c|c|c|c|}
\hline Hole & Core & Section & $\begin{array}{l}\text { Top Interval in } \\
\text { Section }(\mathrm{cm})\end{array}$ & $\begin{array}{l}\text { Depth in } \\
\text { Hole (m) }\end{array}$ & $\begin{array}{l}\text { Total Carbon } \\
\text { Per Cent }\end{array}$ & $\begin{array}{l}\text { Organic Carbon } \\
\text { Per Cent }\end{array}$ & $\begin{array}{l}\mathrm{CaCO}_{3} \\
\text { Per Cent }\end{array}$ \\
\hline 83 & 7 & 2 & 75.0 & 224.1 & $* 8.4$ & $* 0.2$ & 69 \\
\hline 83 & 7 & 3 & 25.0 & 225.1 & $* 7.4$ & $* 0.2$ & 60 \\
\hline 83 & 7 & 4 & 16.0 & 226.5 & 5.9 & 0.2 & 48 \\
\hline 83 & 7 & 5 & 16.0 & 228.0 & 4.7 & 0.2 & 38 \\
\hline 83 & 7 & 6 & 16.0 & 229.5 & 3.8 & 0.3 & 29 \\
\hline 83 & 7 & 6 & 139.0 & 230.7 & 5.3 & 0.3 & 42 \\
\hline 83 & 8 & 7 & 0.0 & 241.9 & 7.7 & 0.1 & 64 \\
\hline $83 \mathrm{~A}$ & 1 & 2 & 31.0 & 14.9 & 7.5 & 0.4 & 60 \\
\hline $83 \mathrm{~A}$ & 1 & 4 & 16.0 & 17.8 & 5.8 & 0.2 & 46 \\
\hline $83 \mathrm{~A}$ & 2 & 1 & 15.0 & 22.3 & $* 5.4$ & $* 0.5$ & 40 \\
\hline $83 \mathrm{~A}$ & 2 & 2 & 16.0 & 23.8 & 6.6 & 0.0 & 55 \\
\hline $83 \mathrm{~A}$ & 2 & 4 & 16.0 & 26.8 & 6.3 & 0.3 & 50 \\
\hline $83 \mathrm{~A}$ & 2 & 5 & 16.0 & 28.3 & $* 7.2$ & $* 0.4$ & 57 \\
\hline $83 \mathrm{~A}$ & 2 & 6 & 16.0 & 29.8 & 7.7 & 0.2 & 63 \\
\hline $83 \mathrm{~A}$ & 3 & 1 & 16.0 & 31.6 & 6.2 & 0.3 & 49 \\
\hline $83 \mathrm{~A}$ & 3 & 2 & 16.0 & 33.1 & 4.5 & 0.6 & 32 \\
\hline $83 \mathrm{~A}$ & 3 & 4 & 16.0 & 36.1 & 4.2 & 0.5 & 31 \\
\hline $83 \mathrm{~A}$ & 3 & 5 & 15.0 & 37.5 & $* 7.9$ & $* 0.2$ & 64 \\
\hline $83 \mathrm{~A}$ & 3 & 6 & 16.0 & 39.1 & 7.2 & 0.3 & 58 \\
\hline $83 \mathrm{~A}$ & 4 & 3 & 15.0 & 43.7 & $* 6.1$ & $* 0.3$ & 49 \\
\hline $83 \mathrm{~A}$ & 4 & 4 & 16.0 & 45.2 & 6.6 & 0.3 & 52 \\
\hline $83 \mathrm{~A}$ & 4 & 6 & 16.0 & 48.2 & 6.9 & 0.3 & 55 \\
\hline $83 \mathrm{~A}$ & 5 & 2 & 30.0 & 51.4 & 7.0 & 0.2 & 57 \\
\hline $83 \mathrm{~A}$ & 5 & 4 & 16.0 & 54.3 & 5.5 & 0.2 & 44 \\
\hline $83 \mathrm{~A}$ & 5 & 5 & 15.0 & 55.8 & $* 5.8$ & $* 0.3$ & 45 \\
\hline $83 \mathrm{~A}$ & 5 & 6 & 16.0 & 57.3 & 5.8 & 0.2 & 47 \\
\hline $83 \mathrm{~A}$ & 6 & 2 & 16.0 & 60.5 & 5.3 & 0.2 & 42 \\
\hline $83 \mathrm{~A}$ & 6 & 4 & 16.0 & 63.5 & 5.8 & 0.3 & 46 \\
\hline $83 \mathrm{~A}$ & 6 & 5 & 15.0 & 64.9 & $* 7.6$ & $* 0.3$ & 61 \\
\hline $83 \mathrm{~A}$ & 6 & 6 & 16.0 & 66.5 & 6.9 & 0.2 & 55 \\
\hline $83 \mathrm{~A}$ & 7 & 1 & 86.0 & 68.8 & 6.2 & 0.2 & 50 \\
\hline $83 \mathrm{~A}$ & 7 & 2 & 16.0 & 69.6 & 6.4 & 0.2 & 52 \\
\hline $83 \mathrm{~A}$ & 7 & 3 & 16.0 & 71.7 & 7.0 & 0.2 & 56 \\
\hline $83 \mathrm{~A}$ & 7 & 4 & 16.0 & 72.6 & 4.3 & 0.3 & 34 \\
\hline $83 \mathrm{~A}$ & 7 & 5 & 15.0 & 74.1 & $* 4.7$ & $* 0.3$ & 36 \\
\hline $83 \mathrm{~A}$ & 7 & 6 & 16.0 & 75.6 & 2.6 & 0.3 & 19 \\
\hline $83 \mathrm{~A}$ & 7 & 6 & 115.0 & 76.6 & $* 5.4$ & $* 0.3$ & 43 \\
\hline $83 \mathrm{~A}$ & 8 & 2 & 16.0 & 78.8 & 6.0 & 0.2 & 48 \\
\hline $83 \mathrm{~A}$ & 8 & 3 & 15.0 & 80.3 & $* 6.9$ & $* 0.2$ & 56 \\
\hline
\end{tabular}


TABLE 1 - Continued

\begin{tabular}{|c|c|c|c|c|c|c|c|}
\hline Hole & Core & Section & $\begin{array}{l}\text { Top Interval in } \\
\text { Section }(\mathrm{cm})\end{array}$ & $\begin{array}{l}\text { Depth in } \\
\text { Hole }(\mathrm{m})\end{array}$ & $\begin{array}{l}\text { Total Carbon } \\
\text { Per Cent }\end{array}$ & $\begin{array}{l}\text { Organic Carbon } \\
\text { Per Cent }\end{array}$ & $\begin{array}{l}\mathrm{CaCO}_{3} \\
\text { Per Cent }\end{array}$ \\
\hline $83 \mathrm{~A}$ & 8 & 4 & 16.0 & 81.8 & 6.7 & 0.2 & 54 \\
\hline $83 \mathrm{~A}$ & 8 & 6 & 16.0 & 84.8 & 6.0 & 0.4 & 47 \\
\hline $83 \mathrm{~A}$ & 8 & 6 & 140.0 & 86.0 & $* 5.8$ & $* 0.2$ & 47 \\
\hline $83 \mathrm{~A}$ & 9 & 1 & 43.0 & 86.5 & *6.6 & $* 0.2$ & 53 \\
\hline $83 \mathrm{~A}$ & 9 & 2 & 16.0 & 87.8 & 2.4 & 0.4 & 17 \\
\hline $83 \mathrm{~A}$ & 9 & 3 & 15.0 & 89.3 & *6.8 & $* 0.3$ & 55 \\
\hline $83 \mathrm{~A}$ & 9 & 4 & 16.0 & 90.8 & 6.5 & 0.3 & 52 \\
\hline $83 \mathrm{~A}$ & 9 & 6 & 16.0 & 93.8 & 5.7 & 0.5 & 43 \\
\hline $83 \mathrm{~A}$ & 10 & 1 & 25.0 & 95.7 & $* 8.7$ & $* 0.2$ & 71 \\
\hline $83 \mathrm{~A}$ & 10 & 2 & 16.0 & 97.1 & 7.8 & 0.3 & 63 \\
\hline $83 \mathrm{~A}$ & 10 & 4 & 16.0 & 100.1 & 8.3 & 0.2 & 68 \\
\hline $83 \mathrm{~A}$ & 10 & 5 & 15.0 & 101.6 & $* 9.0$ & $* 0.2$ & 73 \\
\hline $83 \mathrm{~A}$ & 10 & 6 & 16.0 & 103.1 & 6.2 & 0.3 & 49 \\
\hline $83 \mathrm{~A}$ & 11 & 2 & 16.0 & 106.2 & 6.1 & 0.3 & 48 \\
\hline $83 \mathrm{~A}$ & 11 & 3 & 15.0 & 107.7 & $* 6.6$ & $* 0.2$ & 54 \\
\hline $83 \mathrm{~A}$ & 11 & 4 & 16.0 & 109.2 & 8.2 & 0.2 & 67 \\
\hline $83 \mathrm{~A}$ & 11 & 5 & 15.0 & 110.7 & $* 6.4$ & $* 0.2$ & 52 \\
\hline $83 \mathrm{~A}$ & 11 & 6 & 16.0 & 112.2 & 6.6 & 0.3 & 52 \\
\hline $83 \mathrm{~A}$ & 12 & 2 & 16.0 & 115.3 & 6.8 & 0.3 & 54 \\
\hline $83 \mathrm{~A}$ & 12 & 3 & 15.0 & 116.8 & *6.4 & $* 0.5$ & 50 \\
\hline $83 \mathrm{~A}$ & 12 & 4 & 16.0 & 118.3 & 4.7 & 0.4 & 36 \\
\hline $83 \mathrm{~A}$ & 12 & 5 & 15.0 & 119.8 & $* 4.9$ & $* 0.5$ & 37 \\
\hline $83 \mathrm{~A}$ & 12 & 6 & 16.0 & 121.3 & 5.8 & 0.2 & 47 \\
\hline $83 \mathrm{~A}$ & 13 & 1 & 25.0 & 123.1 & $* 6.3$ & $* 0.3$ & 50 \\
\hline $83 \mathrm{~A}$ & 13 & 2 & 16.0 & 124.5 & 8.0 & 0.2 & 65 \\
\hline $83 \mathrm{~A}$ & 13 & 4 & 16.0 & 127.5 & 8.2 & 0.1 & 68 \\
\hline $83 \mathrm{~A}$ & 13 & 6 & 16.0 & 130.5 & 8.0 & 0.1 & 66 \\
\hline $83 \mathrm{~A}$ & 14 & 1 & 114.0 & 159.2 & $* 6.2$ & $* 0.3$ & 50 \\
\hline $83 \mathrm{~A}$ & 14 & 2 & 16.0 & 159.8 & 7.2 & 0.2 & 58 \\
\hline $83 \mathrm{~A}$ & 14 & 4 & 16.0 & 162.8 & 7.0 & 0.1 & 57 \\
\hline $83 \mathrm{~A}$ & 14 & 5 & 74.0 & 164.8 & $* 7,0$ & $* 0.2$ & 57 \\
\hline $83 \mathrm{~A}$ & 14 & 6 & 16.0 & 165.8 & 7.7 & $* 0.3$ & 61 \\
\hline $83 \mathrm{~A}$ & 15 & 1 & 25.0 & 179.8 & *7.4 & $* 0.2$ & 60 \\
\hline $83 \mathrm{~A}$ & 15 & 2 & 16.0 & 181.2 & 7.3 & $* 0.2$ & 60 \\
\hline $83 \mathrm{~A}$ & 15 & 3 & 75.0 & 183.3 & $* 7.5$ & $* 0.2$ & 61 \\
\hline $83 \mathrm{~A}$ & 15 & 4 & 16.0 & 184.2 & 11.6 & $* 0.0$ & 96 \\
\hline $83 \mathrm{~A}$ & 15 & 5 & 75.0 & 186.3 & $* 7.0$ & $* 0.2$ & 57 \\
\hline $83 \mathrm{~A}$ & 15 & 6 & 16.0 & 187.2 & 6.7 & $* 0.2$ & 54 \\
\hline $83 \mathrm{~A}$ & 16 & 2 & 16.0 & 212.8 & 8.7 & $* 0.1$ & 71 \\
\hline
\end{tabular}


TABLE 1 - Continued

\begin{tabular}{|c|c|c|c|c|c|c|c|}
\hline Hole & Core & Section & $\begin{array}{l}\text { Top Interval in } \\
\text { Section }(\mathrm{cm})\end{array}$ & $\begin{array}{l}\text { Depth in } \\
\text { Hole (m) }\end{array}$ & $\begin{array}{l}\text { Total Carbon } \\
\text { Per Cent }\end{array}$ & $\begin{array}{l}\text { Organic Carbon } \\
\text { Per Cent }\end{array}$ & $\begin{array}{l}\mathrm{CaCO}_{3} \\
\text { Per Cent }\end{array}$ \\
\hline $83 \mathrm{~A}$ & 16 & 3 & 14.0 & 214.2 & $* 8.2$ & $* 0.2$ & 67 \\
\hline $83 \mathrm{~A}$ & 16 & 4 & 16.0 & 215.8 & 8.6 & $* 0.1$ & 70 \\
\hline $83 \mathrm{~A}$ & 16 & 5 & 15.0 & 217.3 & $* 9.0$ & $* 0.1$ & 74 \\
\hline $83 \mathrm{~A}$ & 16 & 6 & 16.0 & 218.8 & 9.0 & $* 0.1$ & 74 \\
\hline 84 & 1 & 1 & 16.0 & 0.2 & 3.1 & $* 1.9$ & 10 \\
\hline 84 & 1 & 2 & 16.0 & 1.7 & 2.8 & $* 1.6$ & 10 \\
\hline 84 & 1 & 4 & 16.0 & 4.7 & 3.2 & $* 1.5$ & 14 \\
\hline 84 & 1 & 6 & 16.0 & 7.7 & 3.4 & $* 1.3$ & 17 \\
\hline 84 & 2 & 2 & 16.0 & 10.9 & 3.1 & $* 1.7$ & 11 \\
\hline 84 & 2 & 4 & 16.0 & 13.9 & 4.2 & $* 1.0$ & 27 \\
\hline 84 & 2 & 6 & 16.0 & 16.9 & 1.9 & $* 1.3$ & 6 \\
\hline 84 & 3 & 2 & 16.0 & 20.0 & 4.9 & $* 1.0$ & 32 \\
\hline 84 & 3 & 4 & 16.0 & 23.0 & 2.0 & $* 1.1$ & 8 \\
\hline 84 & 3 & 6 & 16.0 & 26.0 & 1.6 & $* 0.9$ & 6 \\
\hline 84 & 4 & 2 & 16.0 & 29.1 & 1.9 & $* 1.0$ & 7 \\
\hline 84 & 4 & 4 & 16.0 & 32.1 & 3.7 & $* 1.1$ & 22 \\
\hline 84 & 4 & 6 & 16.0 & 35.1 & 2.2 & $* 0.7$ & 13 \\
\hline 84 & 5 & 2 & 16.0 & 38.3 & 2.4 & $* 0.8$ & 13 \\
\hline 84 & 5 & 4 & 16.0 & 41.3 & 4.8 & $* 1.0$ & 32 \\
\hline 84 & 5 & 6 & 16.0 & 44.3 & 5.0 & *1.0 & 33 \\
\hline 84 & 6 & 2 & 16.0 & 47.4 & 2.8 & $* 1.2$ & 13 \\
\hline 84 & 6 & 4 & 16.0 & 50.4 & 6.9 & $* 0.9$ & 50 \\
\hline 84 & 7 & 2 & 16.0 & 56.6 & 3.4 & *0.9 & 21 \\
\hline 84 & 7 & 4 & 16.0 & 59.6 & 2.6 & $* 1.1$ & 13 \\
\hline 84 & 7 & 6 & 16.0 & 62.6 & 3.0 & $* 1.0$ & 17 \\
\hline 84 & 8 & 2 & 16.0 & 65.7 & 3.8 & $* 0.9$ & 24 \\
\hline 84 & 8 & 4 & 16.0 & 68.7 & 4.0 & $* 1.8$ & 19 \\
\hline 84 & 8 & 5 & 16.0 & 70.2 & 4.9 & *0.7 & 35 \\
\hline 84 & 8 & 6 & 16.0 & 71.7 & 2.5 & *1.0 & 12 \\
\hline 84 & 9 & 2 & 16.0 & 74.9 & 2.8 & $* 1.7$ & 9 \\
\hline 84 & 9 & 4 & 16.0 & 77.9 & 2.2 & $* 1.1$ & 9 \\
\hline 84 & 9 & 6 & 16.0 & 80.9 & 2.4 & $* 1.2$ & 11 \\
\hline 84 & 10 & 2 & 0.0 & 83.8 & 2.6 & *1.1 & 12 \\
\hline 84 & 10 & 4 & 0.0 & 86.8 & 2.6 & *1.1 & 13 \\
\hline 84 & 10 & 6. & 0.0 & 89.8 & 4.1 & $* 1.2$ & 24 \\
\hline 84 & 11 & 2 & 16.0 & 93.1 & 4.1 & ${ }^{*} 0.6$ & 28 \\
\hline 84 & 11 & 4 & 16.0 & 96.1 & 3.7 & *0.6 & 25 \\
\hline 84 & 11 & 6 & 16.0 & 99.1 & 5.5 & $* 0.5$ & 41 \\
\hline
\end{tabular}


TABLE 1 - Continued

\begin{tabular}{|c|c|c|c|c|c|c|c|}
\hline Hole & Core & Section & $\begin{array}{l}\text { Top Interval in } \\
\text { Section }(\mathrm{cm})\end{array}$ & $\begin{array}{l}\text { Depth in } \\
\text { Hole (m) }\end{array}$ & $\begin{array}{c}\text { Total Carbon } \\
\text { Per Cent }\end{array}$ & $\begin{array}{l}\text { Organic Carbon } \\
\text { Per Cent }\end{array}$ & $\begin{array}{l}\mathrm{CaCO}_{3} \\
\text { Per Cent }\end{array}$ \\
\hline 84 & 12 & 2 & 16.0 & 102.3 & 2.1 & $* 0.7$ & 11 \\
\hline 84 & 12 & 4 & 16.0 & 105.3 & 4.9 & 1.1 & 32 \\
\hline 84 & 12 & 6 & 16.0 & 108.3 & 4.6 & $* 0.6$ & 34 \\
\hline 84 & 13 & 2 & 16.0 & 111.4 & 5.4 & $* 0.5$ & 41 \\
\hline 84 & 13 & 4 & 16.0 & 114.4 & 5.6 & $* 0.5$ & 43 \\
\hline 84 & 13 & 6 & 16.0 & 117.4 & 5.5 & $* 0.5$ & 42 \\
\hline 84 & 14 & 2 & 16.0 & 120.6 & 4.4 & $* 0.6$ & 31 \\
\hline 84 & 14 & 4 & 16.0 & 123.6 & 4.4 & $* 0.5$ & 32 \\
\hline 84 & 14 & 6 & 16.0 & 126.6 & 5.1 & $* 0.6$ & 38 \\
\hline 84 & 16 & 2 & 0.0 & 138.7 & 7.4 & $* 0.3$ & 60 \\
\hline 84 & 16 & 4 & 0.0 & 141.7 & 7.7 & $* 0.2$ & 63 \\
\hline 84 & 16 & 6 & 0.0 & 144.7 & 7.4 & *0.3 & 59 \\
\hline 84 & 17 & 2 & 16.0 & 148.0 & 7.1 & $* 0.2$ & 58 \\
\hline 84 & 18 & 2 & 16.0 & 157.2 & 8.3 & $* 0.3$ & 67 \\
\hline 84 & 18 & 4 & 16.0 & 160.2 & 7.4 & $* 0.2$ & 60 \\
\hline 84 & 19 & 4 & 16.0 & 169.3 & 8.8 & $* 0.2$ & 72 \\
\hline 84 & 19 & 6 & 16.0 & 172.3 & 8.4 & $* 0.3$ & 68 \\
\hline 84 & 20 & 2 & 16.0 & 175.4 & 6.7 & $* 0.2$ & 54 \\
\hline 84 & 21 & 5 & 16.0 & 189.1 & 8.8 & $* 0.2$ & 71 \\
\hline 84 & 22 & 2 & 32.0 & 193.8 & 4.0 & $*_{0} .8$ & 27 \\
\hline 84 & 22 & 6 & 16.0 & 199.7 & 8.5 & $* 0.4$ & 67 \\
\hline 84 & 23 & 4 & 16.0 & 205.9 & 7.8 & 0.6 & 60 \\
\hline 84 & 24 & 2 & 16.0 & 212.0 & 7.9 & $* 0.4$ & 62 \\
\hline 84 & 24 & 4 & 16.0 & 215.0 & 8.7 & $* 0.3$ & 70 \\
\hline 84 & 25 & 2 & 16.0 & 221.2 & 8.1 & $* 0.2$ & 65 \\
\hline 84 & 26 & 4 & 16.0 & 233.3 & 9.0 & $* 0.1$ & 74 \\
\hline 84 & 26 & 6 & 16.0 & 236.3 & 10.1 & $* 0.1$ & 84 \\
\hline 84 & 27 & 2 & 16.0 & 239.5 & 8.9 & $* 0.1$ & 73 \\
\hline 84 & 27 & 5 & 16.0 & 244.0 & 8.2 & $* 0.3$ & 66 \\
\hline 84 & 27 & 6 & 16.0 & 245.5 & 8.9 & 0.6 & 69 \\
\hline 84 & 28 & 2 & 16.0 & 248.6 & 7.4 & $* 0.2$ & 61 \\
\hline 84 & 28 & 4 & 16.0 & 251.6 & 7.7 & $* 0.2$ & 62 \\
\hline
\end{tabular}


Precision of these data are as follows:

$$
\begin{aligned}
\text { Total carbon } & \\
\quad(1.2 \text { to } 12 \text { per cent }) & = \pm 0.3 \% \text { absolute } \\
\quad(0 \text { to } 1.2 \text { per cent }) & = \pm 0.06 \% \text { absolute } \\
\text { Organic carbon } & = \pm 0.06 \% \text { absolute } \\
\text { Calcium carbonate } & \\
\quad(10 \text { to } 100 \text { per cent }) & = \pm 3.0 \text { absolute } \\
\quad(0 \text { to } 10 \text { per cent) } & = \pm 1.0 \% \text { absolute }
\end{aligned}
$$

The above methods, precision, and accuracy apply only to Leg 9 data. Values of zero per cent carbon or zero per cent calcium carbonate are not absolute and are obviously subject to our resolution or precision.

\section{ACKNOWLEDGEMENTS}

Special appreciation is acknowledged for the courteous suggestions and assistance of Mary F. Franklin and Tj. H. van Andel of Oregon State University, and H. Mitchell Schwartz of the Laboratory Equipment Corporation, St. Joseph, Michigan.

These samples were analyzed for carbon by G. W. Bode, D. L. Bos, C. E. Chandler, R. H. DuBois, J. B. Isaacs, S. D. Jones, R. J. Minteer, R. E. Olivas and V. S. Sotelo.
Special assistance was provided by R. H. DuBois in calibrating the LECO 70 second analyzer and acid-base system. Computer programming was carried out under the direction of P. B. Woodbury and P. L. Capps.

\section{REFERENCES}

Franklin, M. F., 1970. A possible solution to the controversy concerning the use of LECO instruments for the determination of carbon, sulfur and nitrogen in sediments, rocks and plant materials. $\mathrm{Am}$. Geophys. Union. Preprint (in press).

Laboratory Equipment Corporation, St. Joseph, Michigan. Instruction Manual for the LECO 70 Second Carbon Analyzer, Form 146C. 1.

Laboratory Equipment Corporation, St. Joseph, Michigan. Instruction and Electronic Data Manual for Operation of LECO Induction Furnaces. Models 521,522 , and 523. LECO PATENTS 2,809,100 and $2,779,581$. Canadian Patent No. 560,386.

Laboratory Equipment Corporation, St. Joseph, Michigan, 1959. Instruction Manual for Operation of LECO Carbon Analyzers....Nos. 572-100, 572700, and 577-100. March, 1959, $27 \mathrm{p}$.

Maxwell, John A., 1968. Chemical analysis. In Rock and Mineral Analysis. New York (Interscience) 27, 584. 